premature infant weighing less than about $1,500 \mathrm{~g}$. Help may be obtained by radiological investigations. They may show free air in the abdominal cavity, ranging from small amounts to massive pneumoperitoneum, and patterns indicating intestinal obstruction. At this stage blood cultures frequently give a positive result, so that there is strong indication for surgical intervention and for antibiotic treatment by a suitable drug, usually one which is effective against Gramnegative organisms.

The most commonly affected site is the terminal ileum, followed by the ascending and transverse colon. Fibrinoid necrosis of the mucosa is succeeded by necrotizing enteritis, ulceration, and pseudomembraneous inflammation, and is complicated at times by perforations and intestinal pneumatosis. True obstruction of the intestinal tract has not been found.

\section{Diagnosis of Amyloidosis}

Primary amyloid is a rare disease and none of its clinical manifestations is pathognomonic. The most common symptoms are weakness, dyspnoea, ankle oedema, and loss of weight, but unexplained congestive cardiac failure or the nephrotic syndrome appearing in an adult should arouse suspicion of the possibility of underlying amyloid degeneration. In secondary amyloidosis the presence of the predisposing disease, such as tuberculosis or rheumatoid arthritis, usually alerts the clinician. In primary amyloidosis no such help is present. It is not surprising, therefore, that until recently most cases were diagnosed only post mortem.

The Congo red test, introduced by $\mathrm{H}$. Bennhold ${ }^{1}$ over 40 years ago, is of comparatively little help. A considerable quantity of amyloid is necessary to bind a significant amount of the dye, and this produces a high proportion of false negative results. Techniques for obtaining biopsy specimens of the liver, kidney, and other organs provide a far more reliable diagnostic procedure but introduce new difficulties. Aspiration biopsy of abdominal viscera carries a small but nevertheless real risk and should not be used if the necessary information is obtainable by safer methods. For this reason E. Calkins and A. S. Cohen ${ }^{2}$ introduced biopsy of the gingiva, while P. A. Green ${ }^{3}$ and his colleagues used fragments of small intestine. Once again, however, the incidence of false negatives was too high to warrant the general adoption of these methods.

The high incidence of amyloidosis in familial Mediterranean fever has led to a considerable interest in amyloid among clinical investigators in Israel, and in $1960 \mathrm{~J}$. Gafni and E. Sohar ${ }^{4}$ claimed that a positive diagnosis could be obtained in $75 \%$ of cases by biopsy of the rectal mucous membrane. This compares very well with their own figures of $87 \%$ and $48 \%$ for renal and hepatic biopsies respectively. The general adoption of this method throughout Israel almost doubled the rate of antemortem diagnosis of amyloid degeneration, so that in 1959 a rate of $74 \%$ was achieved, compared with $39 \%$ for the previous eight years.

R. A. Kyle, R. J. Spencer, and D. C. Dahlin ${ }^{5}$ have recently drawn further attention to the value of rectal biopsy in the

\footnotetext{
1 Bennhold, H., Disch. Arch. klin. Med., 1923, 142, 32.

Calkins, E., and Cohen, A. S., Bull. rheum. Dis., 1960, 10, 215.

- Green, P. A., Higgins, J. A., Brown, A. L., iun., Hoffman, H. N., and Sommerville, R. L., Gastroenterology, 1961, 41, 452.

4 Gafn, J., and Sohar, E., Amer. F. med. Sci., 1960, 240, 332.

- Kyle, R. A., Spencer, R. J., and Dahlin, D. C., ibid, 1966, 251, 501
}

diagnosis of primary amyloid. In 20 cases at the Mayo Clinic a definite diagnosis was made by rectal biopsy in 17, and of the 3 false negative results 2 were attributable to an inadequate biopsy specimen. Apparently the submucosa must be included in the specimen, for on 13 occasions the deposits of amyloid were found only in that layer or in the muscularis mucosae and not in the mucous membrane itself. In 79 cases suspected of primary amyloidosis on clinical grounds rectal biopsy was negative. Not one of these has so far shown evidence of the disease, though some have died and necropsy has been carried out, others have had biopsy examinations of other organs, and many have been followed up for several years. This convincing confirmation of the value of rectal biopsy must now make it the method of choice in all patients suspected of having primary amyloidosis.

\section{Medicine in the Tropics}

The practice of medicine in tropical countries differs from that in Britain in many ways. A doctor going out to Africa, for instance, needs to do more than learn the features of malaria and sleeping-sickness. The differential diagnosis of common conditions such as heart failure and abdominal pain requires a reorientation of thought: "common diseases commonly occur," but there is wide variation in the diseases that are common in different countries and among different races. At page 1119 of this week's B.M.F. appears the first of a series of articles on medicine in the tropics. Others will be published at intervals of about a month. These articles will describe diseases and syndromes as seen in the tropics rather than tropical diseases as seen in Britain. The series should help doctors working in tropical countries and particularly those who are working single-handed or on isolated stations. The articles will also be of interest to doctors in Britain who are contemplating work in the developing countries. Besides this, B. Maegraith ${ }^{1}$ has recently drawn attention to the "shrinking world," the result of modern travel, in which there is no exotic disease that may not present in practice in Britain either in a traveller or in an immigrant. The world can now be encircled easily within the incubation period of most of the quarantinable diseases. $^{2}$

In the articles the emphasis will be on practical guidance on diagnosis and management. All clinical investigations and laboratory procedures mentioned will be described in enough detail for a doctor to carry them out without skilled assistance. Treatment will also be described in detail, the dosage and duration of administration of drugs will be stated, and the problems that arise in the management of patients in underdeveloped rural communities will be discussed.

For centuries British doctors have studied and practised tropical medicine. Maegraith's shrinking world is making practical knowledge of tropical disease more and more essential for all doctors, wherever they work. The articles in this series are written by experts with first-hand knowledge of their subjects, but each one will be describing disease as it occurs in only one portion of the world. We hope that this series will stimulate interest in this challenging field, and perhaps provoke correspondence from others with different experience. 